\title{
Sober topological spaces valued in a quantale
}

\author{
Dexue Zhang, Gao Zhang \\ dxzhang@scu.edu.cn, gaozhang0810@hotmail.com
}

\begin{abstract}
The notion of sobriety is extended to the realm of topological spaces valued in a commutative and unital quantale, via an adjunction between a category of quantale modules and the category of quantale-valued topological spaces. Relations between such sober spaces and quantale-valued domains based on flat ideals are investigated.

Keywords Fuzzy topology; Q-order; Q-module; Flat ideal; Sober Q-topological space MSC(2020) 54A40 54B30 18B30
\end{abstract}

\section{Introduction}

Sobriety is an interesting topological property for non-Hausdorff spaces and plays an important role in domain theory [3, 4]. A main feature of such a space is that the space can be recovered from its open set lattice. Sobriety is often described via the adjunction

$$
\mathcal{O} \dashv \mathrm{pt}: \mathrm{Frm}^{\mathrm{op}} \longrightarrow \text { Top }
$$

between the opposite of the category of frames and the category of topological spaces [10]. A topological space $X$ is sober if the unit $\eta_{X}: X \longrightarrow \operatorname{pt} \mathcal{O}(X)$ is a bijection (hence a homeomorphism).

Different extensions of the notion of sobriety to the fuzzy context have been proposed since 1990. Here we mention a few of the works on this topic: Rodabaugh [26], Zhang and Liu [42, Kotzé [14, Srivastava and Khastgir [30], Pultr and Rodabaugh [24, 25], Gutiérrez García, Höhle and de Prada Vicente [5], Yao [37, 38], Jäger and Yao [9], Singh and Srivastava [29], and etc. In these works, the table of truth-values is assumed to be a complete Heyting algebra (i.e., a frame), sometimes even a completely distributive lattice. For such a table of truth-values, the logic connective conjunction, modeled by the meet operation of the lattice, is idempotent. From the viewpoint of fuzzy logic, this is a serious drawback. For example, BL-algebras [6] and left-continuous t-norms [13] are seldom idempotent.

The aim of this paper is to extend the notion of sobriety to the realm of topological spaces valued in a commutative and unital quantale. Complete Heyting algebras, BLalgebras, and the interval $[0,1]$ equipped with a left-continuous t-norm are typical examples of such quantales.

This paper is a companion of [41], which focuses on sobriety of quantale-valued cotopological spaces. In the classical context, a topological space can be described by open sets as well as by closed sets, and we can switch between open sets and closed sets by taking complements. Thus, it makes no difference whether we choose to work with closed sets or with open sets in the classical context. But, in the fuzzy context, the table of truth-values usually does not satisfy the law of double negation, there is no natural way 
to switch between open sets and closed sets, so, as observed in 41], in fuzzy topology it is often necessary to consider the topology version (in term of open sets) and the cotopology version (in term of closed sets) of the same concept. In other words, like a coin, fuzzy topology was born with two sides. And, we would like to stress that, due to the lack of symmetry in the table of truth-values, different ideas and techniques are needed in the topology and the cotopology versions of the same theory.

In this paper, like that in [41], we emphasize the connection between quantale-valued orders and quantale-valued topologies. For each commutative and unital quantale Q, a category of Q-modules (or equivalently, complete Q-lattices) is specified, sober Q-topological spaces are then described via an adjunction between this category and that of Q-topological spaces. It is shown that for a commutative and integral quantale, the Scott Q-topology of every $\mathcal{F}$-domain (see Definition 4.5 ) is sober. In the final section, some examples are presented in the case that the quantale is the interval $[0,1]$ together with a continuous t-norm.

\section{Quantales and quantale-valued orders}

In this section we recall some basic ideas about quantale-valued orders and fix some notations.

A frame [10] is a complete lattice $L$ such that the binary meet operation $\wedge$ distributes over arbitrary joins; that is, $x \wedge \bigvee S=\bigvee\{x \wedge s \mid s \in S\}$ for each $x \in L$ and each $S \subseteq L$. A frame map $f: L_{1} \longrightarrow L_{2}$ between frames is a map that preserves arbitrary joins and finite meets, including the empty ones. Frames and frame maps constitute a category

\section{Frm.}

Following Rodabaugh [26, page 303], we say that a map $f: L \longrightarrow M$ between complete lattices (not necessarily frames) is frame-like if $f$ preserves arbitrary joins and finite meets.

A quantale [27] $Q=(Q, \&)$ is a semigroup such that the underlying set $Q$ is a complete lattice and that the multiplication \& distributes over arbitrary joins on both sides. As usual, we write 0 and 1 for the bottom and the top element of $Q$, respectively. A quantale $Q$ is

- commutative if the multiplication \& is commutative;

- unital if it has a unit element $k$;

- integral if it is unital and the unit $k$ is the top element of Q.

It is clear that a complete lattice $L$ is a frame if, and only if, $(L, \wedge)$ is a quantale. In this paper, when we say that a quantale $Q=(Q, \&)$ is a frame, we mean the semigroup operation \& of $Q$ is the meet operation $\wedge$. It is possible that the underlying lattice of a quantale is a frame, but the quantale itself is not a frame.

Standing Assumption. In this paper, by a quantale we always mean a commutative and unital one, with unit denoted by $k$, unless otherwise specified.

Given a quantale $\mathrm{Q}$, the multiplication \& determines a binary operator $\rightarrow$, known as the implication operator of $\&$, via the adjoint property:

$$
p \& q \leq r \Longleftrightarrow q \leq p \rightarrow r .
$$


A Q-valued order, a Q-order for short, is a map $\alpha: X \times X \longrightarrow \mathrm{Q}$ such that for all $x, y, z \in X$,

$$
\alpha(x, x) \geq k \quad \text { and } \quad \alpha(y, z) \& \alpha(x, y) \leq \alpha(x, z) .
$$

The pair $(X, \alpha)$ is call a $\mathrm{Q}$-ordered set, or a $\mathrm{Q}$-category in the language of enriched categories [20]. It is customary to write $X$ for the pair $(X, \alpha)$ and write $X(x, y)$ for $\alpha(x, y)$.

A map $f: X \longrightarrow Y$ between Q-ordered sets is said to preserve Q-order if $X(x, y) \leq$ $Y(f(x), f(y))$ for all $x, y \in X$. Q-ordered sets and Q-order-preserving maps constitute a category

Q-Ord.

If $\alpha$ is a $\mathrm{Q}$-order on $X$, it follows from the commutativity of \& that $\alpha^{\mathrm{op}}(x, y):=\alpha(y, x)$ is also a $\mathrm{Q}$-order on $X$, called the opposite of $\alpha$.

The following two examples belong to the folklore in the theory of Q-orders, it is hard to specify where they appeared for the first time.

Example 2.1. For all $x, y \in \mathrm{Q}$, let

$$
\alpha_{L}(x, y)=x \rightarrow y \text {. }
$$

Then $\alpha_{L}$ is a Q-order, called the canonical Q-order, on Q. The opposite of $\alpha_{L}$ is denoted by $\alpha_{R}$; that is,

$$
\alpha_{R}(x, y)=y \rightarrow x .
$$

Example 2.2. For each set $X$, the map

$$
\operatorname{sub}_{X}: \mathrm{Q}^{X} \times \mathrm{Q}^{X} \longrightarrow \mathrm{Q}, \quad \operatorname{sub}_{X}(\phi, \psi)=\bigwedge_{x \in X} \phi(x) \rightarrow \psi(x)
$$

is a $\mathrm{Q}$-order on the set $\mathrm{Q}^{X}$, known as the inclusion $\mathrm{Q}$-order. If $X$ is a singleton set, then $\left(X, \operatorname{sub}_{X}\right)$ degenerates to the $\mathrm{Q}$-ordered set $\left(\mathrm{Q}, \alpha_{L}\right)$.

The underlying order of a $Q$-ordered set $X$ refers to the order $\leq$ given by

$$
x \leq y \quad \text { if } k \leq X(x, y) .
$$

For convenience, for each $\mathrm{Q}$-ordered set $X$, we shall write $X_{0}$ for $X$ with the underlying order.

Two elements $x, y$ of a Q-ordered set $X$ are isomorphic if $k \leq X(x, y) \wedge X(y, x)$. A Q-ordered set $X$ is separated if isomorphic elements are identical; that is,

$$
k \leq X(x, y) \wedge X(y, x) \Longrightarrow x=y .
$$

Let $X, Y$ be Q-ordered sets; let $f: X \longrightarrow Y$ and $g: Y \longrightarrow X$ be maps. We say that $f$ is left adjoint to $g$ (and/or, $g$ is right adjoint to $f$ ) and write $f \dashv g$, if

$$
Y(f(x), y)=X(x, g(y))
$$

for all $x \in X$ and $y \in Y$. This is a special case of enriched adjunctions in category theory [1, 20].

Theorem 2.3. ([32, page 295]) Let $f: X \longrightarrow Y$ and $g: Y \longrightarrow X$ be a pair of maps between $Q$-ordered sets. Then $f$ is left adjoint to $g$ if, and only if, the following conditions are satisfied: 
(i) Both $f$ and $g$ preserve Q-order.

(ii) The map $f: X_{0} \longrightarrow Y_{0}$ is left adjoint to $g: Y_{0} \longrightarrow X_{0}$; that is, for all $x \in X$ and $y \in Y, f(x) \leq y \Longleftrightarrow x \leq g(y)$.

Let $X$ be a $\mathrm{Q}$-ordered set. A weight of $X$ is a map $\phi: X \longrightarrow \mathrm{Q}$ such that for all $x, y \in X$,

$$
\phi(y) \& X(x, y) \leq \phi(x) .
$$

Remark 2.4. There exist different terminologies for a weight of a $Q$-ordered set $X$. Firstly, since the above inequality can be read as "that $y$ belongs to $\phi$ and $x$ is smaller than or equal to $y$ implies $y$ belongs to $\phi "$, a weight is also called a lower fuzzy set of $X$. Secondly, since a weight of $X$ is just a $Q$-order-preserving map (or, a $Q$-functor) $\phi: X^{\text {op }} \longrightarrow\left(\mathrm{Q}, \alpha_{L}\right.$ ), it is also called a $\mathrm{Q}$-presheaf of $X$ (viewed as an enriched category). The terminology weight adopted here comes from category theory, see e.g. [12].

The weights of a $\mathrm{Q}$-ordered set $X$ constitute a $\mathrm{Q}$-ordered set $\mathcal{P} X$ with

$$
\mathcal{P} X\left(\phi_{1}, \phi_{2}\right):=\operatorname{sub}_{X}\left(\phi_{1}, \phi_{2}\right) .
$$

For each $x \in X, X(-, x)$ is a weight of $X$ and we have the following:

Lemma 2.5 (Yoneda lemma). Let $X$ be a Q-ordered set and $\phi$ be a weight of $X$, then

$$
\mathcal{P} X(X(-, x), \phi)=\phi(x) .
$$

The Yoneda lemma entails that the map

$$
\mathrm{y}_{X}: X \longrightarrow \mathcal{P} X, \quad x \mapsto X(-, x)
$$

is an embedding if $X$ is separated. By abuse of language, we call it the Yoneda embedding no matter $X$ is separated or not.

Each Q-order-preserving map $f: X \longrightarrow Y$ gives rise to a natural adjunction between $\mathcal{P} X$ and $\mathcal{P} Y$. Precisely, the map

$$
f^{\rightarrow}: \mathcal{P} X \longrightarrow \mathcal{P} Y, \quad f^{\rightarrow}(\phi)(y)=\bigvee_{x \in X} \phi(x) \& Y(y, f(x))
$$

is left adjoint to

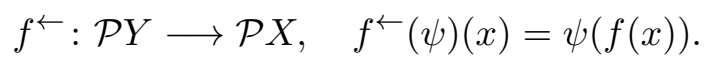

Let $X$ be a Q-ordered set and let $\phi$ be a weight of $X$. We say that an element $a$ of $X$ is a supremum of $\phi$ and write $a=\sup \phi$, if

$$
X(a, y)=\mathcal{P} X(\phi, X(-, y))
$$

for all $y \in X$. In the language of category theory, $\sup \phi$ is the colimit of the identity functor id: $X \longrightarrow X$ weighted by $\phi$ (see e.g. [1, 31]). Suprema of a weight, if exist, are unique up to isomorphism.

We say that a $\mathrm{Q}$-ordered set $X$ is cocomplete if every weight of $X$ has a supremum. It is clear that $X$ is cocomplete if, and only if, the Yoneda embedding $\mathrm{y}_{X}: X \longrightarrow \mathcal{P} X$ has a left adjoint (see e.g. [31]). 
Example 2.6. The $\mathrm{Q}$-ordered set $\left(\mathrm{Q}, \alpha_{L}\right)$ is cocomplete. For each weight $\phi$ of $\left(\mathrm{Q}, \alpha_{L}\right)$,

$$
\sup \phi=\bigvee_{x \in Q} \phi(x) \& x=\phi(k) .
$$

A coweight of a $\mathrm{Q}$-ordered set $X$ is defined to be weight of the opposite $X^{\text {op }}$ of $X$. Explicitly, a coweight of $X$ is a $\mathrm{Q}$-order-preserving map $\psi: X \longrightarrow\left(\mathrm{Q}, \alpha_{L}\right)$. Coweights are also known as upper fuzzy sets and covariant $\mathrm{Q}$-presheaves. The coweights of $X$ constitute a Q-ordered set $\mathcal{P}^{\dagger} X$ with

$$
\mathcal{P}^{\dagger} X\left(\psi_{1}, \psi_{2}\right):=\operatorname{sub}_{X}\left(\psi_{2}, \psi_{1}\right)
$$

Let $\psi$ be a coweight and let $a$ be an element of $X$. We say that $a$ is an infimum of $\psi$ if

$$
X(y, a)=\mathcal{P}^{\dagger} X(X(y,-), \psi)
$$

for all $y \in X$.

A Q-ordered set $X$ is complete if every coweight of $X$ has an infimum. It is known [31] that

(i) a Q-ordered set $X$ is cocomplete if, and only if, $X$ is complete; and

(ii) a Q-order-preserving map $f: X \longrightarrow Y$ between cocomplete Q-ordered sets is a left adjoint if, and only if, $f$ preserves suprema in the sense that $f(\sup \phi)=\sup f^{\rightarrow}(\phi)$ for each weight $\phi$ of $X$.

A Q-ordered set is a complete Q-lattice if $X$ is separated and complete (or equivalently, cocomplete). Complete Q-lattices and left adjoints constitute a category

$$
\text { Q-Sup. }
$$

For each Q-ordered set $X, \mathcal{P} X$ is cocomplete, hence a complete Q-lattice [31]. Actually,

$$
\mathrm{y}_{X}^{\overleftarrow{X}}: \mathcal{P} \mathcal{P} X \longrightarrow \mathcal{P} X
$$

is a left adjoint of $\mathrm{y}_{\mathcal{P} X}: \mathcal{P} X \longrightarrow \mathcal{P} \mathcal{P} X$. This means that the supremum of a weight $\Phi$ of $\mathcal{P} X$ is given by

$$
\sup \Phi=\mathrm{y}_{X}^{\overleftarrow{X}}(\Phi)=\bigvee_{\phi \in \mathcal{P} X} \Phi(\phi) \& \phi
$$

The correspondence

$$
f: X \longrightarrow Y \quad \mapsto \quad f^{\rightarrow}: \mathcal{P} X \longrightarrow \mathcal{P} Y
$$

defines a functor $\mathcal{P}:$ Q-Ord $\longrightarrow$ Q-Sup that is left adjoint to the forgetful functor Q-Sup $\longrightarrow$ Q-Ord [31]. We write

$$
\mathbb{P}=(\mathcal{P}, \mathrm{m}, \mathrm{y})
$$

for the monad on Q-Ord arising from this adjunction. Explicitly,

- for each Q-order-preserving map $f: X \longrightarrow Y, \mathcal{P} f=f \rightarrow: \mathcal{P} X \longrightarrow \mathcal{P} Y$;

- the unit is the Yoneda embedding $\mathrm{y}_{X}: X \longrightarrow \mathcal{P} X$;

- the multiplication $\mathrm{m}_{X}=\sup _{\mathcal{P} X}=\mathrm{y}_{X}^{\overleftarrow{X}}: \mathcal{P} \mathcal{P} X \longrightarrow \mathcal{P} X$ 
The category of $\mathbb{P}$-algebras and $\mathbb{P}$-homomorphisms is precisely that of Q-complete lattices and left adjoints (see e.g. [18, 31]); that is,

$$
\mathbb{P}-\mathrm{Alg}=\mathrm{Q}-\text { Sup. }
$$

As we see below, there is a very useful and convenient characterization of Q-Sup, it is equivalent to the category of Q-modules. Recall that the category Sup of complete lattices and join-preserving maps is symmetric and monoidal closed, see [11, Chapter I], thus, as in any such categories [22, one can talk about monoids and monoid actions in Sup. It is readily seen that a commutative and unital quantale $Q$ is exactly a commutative monoid in Sup. So, there are Q-actions and Q-modules.

Definition 2.7. ([11]) A Q-module (precisely, a left Q-module) is a pair $(X, \otimes)$, where $X$ is a complete lattice and $\otimes: \mathrm{Q} \times X \longrightarrow X$ is a map, called a (left) Q-action on $X$, subject to the following conditions: for all $x \in X$ and $r, s, \in \mathrm{Q}$,

(i) $k \otimes x=x$, where $k$ is the unit of $\mathrm{Q}$;

(ii) $s \otimes(r \otimes x)=(s \& r) \otimes x$;

(iii) $r \otimes-: X \longrightarrow X$ preserve joins; and

(iv) $-\otimes x: \mathrm{Q} \longrightarrow X$ preserve joins.

The conditions (iii) and (iv) together amount to requiring that $\otimes: \mathrm{Q} \times X \longrightarrow X$ is a bimorphism in Sup (see e.g. [11, page 5]). Thus, by universal property of tensor products in Sup, one sees that the above definition of Q-modules, which is a bit more reader-friendly, is equivalent to that in [11].

A homomorphism $f:(X, \otimes) \longrightarrow(Y, \otimes)$ between Q-modules is a join-preserving map $f: X \longrightarrow Y$ that preserves the action, i.e., $r \otimes f(x)=f(r \otimes x)$ for all $r \in \mathrm{Q}$ and $x \in X$. Q-modules and homomorphisms constitute a category

\section{Q-Mod.}

If $\mathrm{Q}$ is the Boolean algebra $(\{0,1\}, \wedge)$, then $\mathrm{Q}-\mathrm{Mod}$ is equivalent to the category Sup of complete lattices and join-preserving maps [11, page 8, Proposition 1].

The quantale $(\mathrm{Q}, \&)$ itself is a $\mathrm{Q}$-module, with \& being the action. We often view $\mathrm{Q}$ as a $\mathrm{Q}$-module in this way.

Let $X$ be a $\mathrm{Q}$-ordered set. For each $x \in X$ and $r \in \mathrm{Q}$, the tensor of $r$ and $x$ (see e.g. [32, page 288]), denoted by $r \otimes x$, is a point of $X$ such that for all $y \in X$,

$$
X(r \otimes x, y)=r \rightarrow X(x, y) .
$$

A Q-ordered set $X$ is tensored if the tensor $r \otimes x$ exists for all $x \in X$ and $r \in \mathrm{Q}$. Some facts about tensors are listed below, which can be found in [32] and [17].

- Every complete Q-lattice is tensored. Actually, $r \otimes x$ is the supremum of the weight $r \& X(-, x)$.

- Every left adjoint $f$ preserves tensors; that is, $r \otimes f(x)=f(r \otimes x)$.

- A map $f: X \longrightarrow Y$ between complete Q-lattices preserves Q-order if, and only if, $f: X_{0} \longrightarrow Y_{0}$ preserves order and $r \otimes f(x)=f(r \otimes x)$ for all $x \in X$ and $r \in \mathrm{Q}$. 
Given a complete Q-lattice $X$, taking tensor $(r, x) \mapsto r \otimes x$ makes $X$ into a Q-module. Conversely, given a $\mathrm{Q}$-module $(X, \otimes)$, if we let

$$
\alpha(x, y)=\bigvee\{r \in \mathrm{Q} \mid r \otimes x \leq y\}
$$

then $(X, \alpha)$ is a complete $\mathrm{Q}$-lattice. These two processes are inverse to each other. So we have the following characterization of Q-Sup, which is an instance of [32, Corollary 4.13].

Proposition 2.8. The category Q-Mod is equivalent to the category Q-Sup.

Example 2.9. For each set $X$, the assignment $(r, \lambda) \mapsto r \& \lambda$ defines a $\mathrm{Q}$-action on $\mathrm{Q}^{X}$, so, $\mathrm{Q}^{X}$ becomes a $\mathrm{Q}$-module. We shall always view $\mathrm{Q}^{X}$ as a $\mathrm{Q}$-module in this way. It is clear that the corresponding $\mathrm{Q}$-order of the $\mathrm{Q}$-module $\mathrm{Q}^{X}$ is just the inclusion $\mathrm{Q}$-order $\operatorname{sub}_{X}$ in Example 2.2.

For more information on Q-modules and their relations to Q-orders the reader is referred to the monograph [2].

\section{Sober Q-topological spaces}

A Q-topology on a set $X$ is subset $\tau$ of $\mathrm{Q}^{X}$ subject to the following conditions:

(O1) The constant map $1_{X}: X \longrightarrow \mathrm{Q}$ with value 1 belongs to $\tau$;

(O2) $\lambda \wedge \mu \in \tau$ for all $\lambda, \mu \in \tau$;

(O3) $\bigvee_{j \in J} \lambda_{j} \in \tau$ for each subset $\left\{\lambda_{j}\right\}_{j \in J}$ of $\tau$;

(O4) $\lambda \& r \in \tau$ for all $r \in \mathrm{Q}$ and $\lambda \in \tau$, where $(\lambda \& r)(x)=\lambda(x) \& r$ for all $x \in X$.

The pair $(X, \tau)$ is called a $\mathrm{Q}$-topological space; elements of $\tau$ are called open sets.

It is customary to write $X$ for a Q-topological space and write $\mathcal{O}(X)$ for the set of open sets of $X$. The interior operator of a Q-topological space $X$ refers to the map

$$
(-)^{\circ}: \mathrm{Q}^{X} \longrightarrow \mathrm{Q}^{X}, \quad \lambda^{\circ}=\bigvee\{\mu \in \mathcal{O}(X) \mid \mu \leq \lambda\}
$$

The interior operator satisfies the following conditions: for all $\lambda, \mu \in \mathrm{Q}^{X}$, (int1) $1_{X}^{\circ}=1_{X}$; (int2) $\lambda^{\circ} \leq \lambda$; (int3) $(\lambda \wedge \mu)^{\circ}=\lambda^{\circ} \wedge \mu^{\circ} ;(\operatorname{int} 4) \operatorname{sub}_{X}(\lambda, \mu) \leq \operatorname{sub}_{X}\left(\lambda^{\circ}, \mu^{\circ}\right)$; and (int5) $\left(\lambda^{\circ}\right)^{\circ}=\lambda^{\circ}$. Actually, Q-topologies on $X$ correspond bijectively to operators $\mathrm{Q}^{X} \longrightarrow \mathrm{Q}^{X}$ satisfying (int1)-(int5). This fact also justifies the definition of Q-topology.

A map $f: X \longrightarrow Y$ between Q-topological spaces is continuous if $\lambda \circ f$ is an open set of $X$ for each open set $\lambda$ of $Y$. The category of Q-topological spaces and continuous maps is denoted by

$$
\text { Q-Top. }
$$

Given a Q-topological space $X$, the Q-relation

$$
\Omega X: X \times X \rightarrow \mathrm{Q}, \quad \Omega X(x, y)=\bigwedge_{\lambda \in \mathcal{O}(X)} \lambda(x) \rightarrow \lambda(y)
$$

is a Q-order, called the specialization $\mathrm{Q}$-order of the space $X$. In this way, we obtain a functor

$$
\Omega: \text { Q-Top } \longrightarrow \text { Q-Ord. }
$$


It is known that $\Omega$ has a left adjoint (see e.g. [15]).

We say that a Q-topological space $X$ is $T_{0}$ if for any pair $x, y$ of distinct points, there is an open set $\lambda$ such that $\lambda(x) \neq \lambda(y)$. It is clear that $X$ is $T_{0}$ if, and only if, the specialization Q-order of $X$ is separated.

Write

$$
\text { Q-Mod }{ }_{\text {frm }}
$$

for the category composed of Q-modules and frame-like Q-module homomorphisms, that is, $Q$-module homomorphisms that preserves finite meets. In the case that $Q$ is the twoelement Boolean algebra, Q-Mod frm $_{\text {frm }}$ coincides with the category CSLF of complete lattices and frame-like maps in Rodabaugh [26].

For each Q-topological space $X$, by commutativity of $\&$, the set $\mathcal{O}(X)$ of open sets of $X$ is a $\mathrm{Q}$-module with $\mathrm{Q}$-action

$$
r \otimes \lambda:=\lambda \& r
$$

The correspondence $X \mapsto \mathcal{O}(X)$ gives rise to a functor

$$
\mathcal{O}: \text { Q-Top } \longrightarrow \text { Q-Mod } \text { frm }_{\text {op }} \text {. }
$$

Given a Q-module $(A, \otimes)$, by a point of $(A, \otimes)$ we mean a frame-like Q-module homomorphism

$$
p:(A, \otimes) \longrightarrow(\mathrm{Q}, \&) .
$$

Explicitly, a point of $(A, \otimes)$ is a map $p: A \longrightarrow \mathrm{Q}$ subject to the following conditions:

(pt1) $p(\top)=1$, where $T$ is the top element of $A$;

$(\mathrm{pt} 2) p(\lambda \wedge \mu)=p(\lambda) \wedge p(\mu)$;

(pt3) $p\left(\bigvee_{i \in I} \lambda_{i}\right)=\bigvee_{i \in I} p\left(\lambda_{i}\right)$;

$(\mathrm{pt} 4) p(r \otimes \lambda)=r \& p(\lambda)$.

Definition 3.1. [41] A Q-topological space $X$ is sober if for each point $p: \mathcal{O}(X) \longrightarrow \mathrm{Q}$ of the Q-module $\mathcal{O}(X)$, there is a unique point $x$ of $X$ such that $p(\lambda)=\lambda(x)$ for all $\lambda \in \mathcal{O}(X)$.

Remark 3.2 (When $Q$ is a frame, $I)$. Assume that $Q=(Q, \&)$ is a frame, i.e., $\&=\wedge$. For each Q-topological space $X$, the assignment $r \mapsto r_{X}$ defines a frame map $i_{X}: \mathrm{Q} \longrightarrow \mathcal{O}(X)$, where $r_{X}$ denotes the constant open set with value $r$. It is clear that a map $p: \mathcal{O}(X) \longrightarrow \mathrm{Q}$ is a point of the Q-module $\mathcal{O}(X)$ if, and only if, $p$ is a frame map such that $p \circ i_{X}$ is the identity map. Thus, for such a quantale, a Q-topological space is sober in the sense of Definition 3.1 if, and only if, it is sober in the sense of [42].

Example 3.3. (See [30, Example 3.2(b)] in the case that $(Q, \&)$ is a frame) Write $\mathbb{S}$ for the Q-topological space obtained by endowing Q with the coarsest Q-topology that has the identity map as an open set. We call $\mathbb{S}$ the Sierpinski Q-topological space. It is readily verified that the specialization $\mathbf{Q}$-order of $\mathbb{S}$ is the canonical Q-order on $\mathbf{Q}$; that is, $\Omega \mathbb{S}=\left(\mathrm{Q}, \alpha_{L}\right)$.

If $(\mathrm{Q}, \&)$ satisfies the following requirements:

- $(\mathrm{Q}, \&)$ is integral,

- the underlying lattice $\mathrm{Q}$ is a frame, 
- $(a \wedge b) \& r=(a \& r) \wedge(b \& r)$ for all $r, a, b \in \mathrm{Q}$,

then, by help of the identity $(a \vee(x \& r)) \wedge b=(a \wedge b) \vee(b \wedge(x \& r))$ one sees that

$$
\mathcal{O}(\mathbb{S})=\{(a \vee(\mathrm{id} \& r)) \wedge b \mid r, a, b \in \mathrm{Q}\} .
$$

Thus, each map $p: \mathcal{O}(\mathbb{S}) \longrightarrow Q$ satisfying (pt1)-(pt4) is determined by its value at the identity map on $\mathbf{Q}$. From this one derives that $p(\lambda)=\lambda(a)$ for all $\lambda \in \mathcal{O}(\mathbb{S})$, where $a=p(\mathrm{id})$, hence $\mathbb{S}$ is sober.

As one expects, sobriety of Q-topological spaces can be described via an adjunction

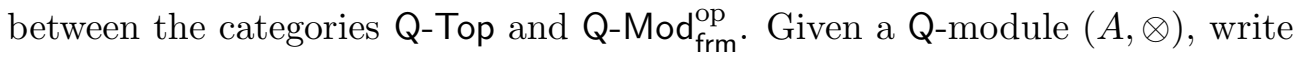

$$
\operatorname{pt} A
$$

for the set of all points of $(A, \otimes)$. For each $\lambda \in A$, define

$$
\widehat{\lambda}: \mathrm{pt} A \longrightarrow \mathrm{Q}
$$

by

$$
\widehat{\lambda}(p)=p(\lambda)
$$

Then

$$
\widehat{A}:=\{\widehat{\lambda} \mid \lambda \in A\}
$$

is a Q-topology on pt $A$ and the assignment

$$
(A, \otimes) \mapsto(\operatorname{pt} A, \widehat{A})
$$

defines a functor

$$
\text { pt: Q-Mod } \text { frm }_{\text {pr }} \longrightarrow \text { Q-Top. }
$$

Proposition 3.4. pt: Q-Mod frm $_{\text {op }}^{\text {p }} \longrightarrow$ Q-Top is right adjoint to $\mathcal{O}: \mathrm{Q}-$ Top $\longrightarrow$ Q-Mod frm $_{\text {fo }}^{\text {. }}$

Proof. We only spell out the unit and the counit of the adjunction here. The unit: for each Q-topological space $X$,

$$
\eta_{X}: X \longrightarrow \operatorname{pt} \mathcal{O}(X), \quad \eta_{X}(x)(\lambda)=\lambda(x) .
$$

The counit: for each $\mathrm{Q}$-module $(A, \otimes)$,

$$
\varepsilon_{A}: A \longrightarrow \mathcal{O}(\operatorname{pt} A), \quad \varepsilon_{A}(\lambda)=\widehat{\lambda} .
$$

Proposition 3.5. Let $X$ be a $\mathrm{Q}$-topological space and let $(A, \otimes)$ be a $\mathrm{Q}$-module.

(i) $X$ is a $T_{0}$ space if, and only if, $\eta_{X}: X \longrightarrow \operatorname{pt} \mathcal{O}(X)$ is injective.

(ii) $X$ is sober if, and only if, $\eta_{X}: X \longrightarrow \operatorname{pt} \mathcal{O}(X)$ is bijective, hence a homeomorphism.

(iii) The Q-topological space pt $A$ is sober. In particular, the space $\mathrm{pt} \mathcal{O}(X)$ is sober and is called the sobrification of $X$.

Proof. The verification is standard. For example, we check that if $\eta_{X}: X \longrightarrow \operatorname{pt} \mathcal{O}(X)$ is bijective, then it is a homeomorphism. For this, we check that $\eta_{X}$ is an open map in this case. Write $\hat{x}$ for the point $\eta_{X}(x)$ of pt $\mathcal{O}(X)$. Since $\eta_{X}$ is bijective, every point of pt $\mathcal{O}(X)$ is of the form $\hat{x}$ for a unique $x \in X$. Since for each open set $\lambda$ and each point $x$ of $X, \hat{\lambda}(\hat{x})=\eta_{X}(x)(\lambda)=\lambda(x)$, it follows that the image of $\lambda$ under $\eta_{X}$ is $\widehat{\lambda}$, hence $\eta_{X}$ is an open map. 
A Q-module $(A, \otimes)$ is spatial if the counit $\varepsilon_{A}: A \longrightarrow \mathcal{O}(\mathrm{pt} A)$ is an isomorphism. It is clear that for each $\mathrm{Q}$-topological space $X$, the $\mathrm{Q}$-module $\mathcal{O}(X)$ is spatial. The category of sober Q-topological spaces is dually equivalent to the subcategory of Q-Mod $\mathrm{d}_{\mathrm{frm}}$ consisting spatial Q-modules, and is a reflective full subcategory of Q-Top.

Remark 3.6 (When $Q$ is a frame, II). Assume that $Q=(Q, \&)$ is a frame, i.e., $\&=\wedge$. Let $i: \mathrm{Q} \longrightarrow A$ be an object of the slice category $\mathrm{Q} \downarrow$ Frm; that is, $i$ is a frame map. Define $\otimes: \mathrm{Q} \times A \longrightarrow A$ by $r \otimes x=x \wedge i(r)$. Then $(A, \otimes)$ is a $\mathrm{Q}$-module. This assignment makes

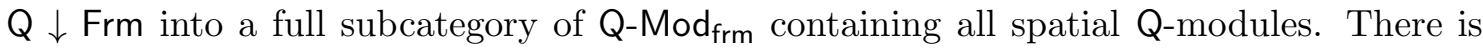
a nice characterization of this subcategory: a Q-module $(A, \otimes)$ is generated by an object in the slice category $\mathrm{Q} \downarrow$ Frm if, and only if, for each $x \in A$ the map $x \wedge-: A \longrightarrow A$ is a Q-module homomorphism. This characterization is essentially Proposition 3.7 and Proposition 3.9 in [38]. For the sake of completeness, a direct verification is included here.

Necessity is obvious, we only need to check the sufficiency. First, since $x \wedge-: A \longrightarrow A$ is a $\mathrm{Q}$-module homomorphism for all $x \in A$, it follows that, in $A$, binary meets distribute over arbitrary joins, hence $A$ is a frame. Define $i: \mathrm{Q} \longrightarrow A$ by $i(r)=r \otimes \top$, where $\top$ is the top element of the frame $A$. We claim that $i$ is a frame map and the Q-module $(A, \otimes)$ is generated by $i$. That $i$ preserves join is clear, to see that it preserves finite meet, let $r, s \in \mathrm{Q}$. Then

$$
\begin{aligned}
i(r) \wedge i(s) & =(r \otimes \top) \wedge(s \otimes \top) \\
& =s \otimes((r \otimes \top) \wedge \top) \\
& =s \otimes(r \otimes \top) \\
& =(s \wedge r) \otimes \top \\
& =i(r \wedge s),
\end{aligned}
$$

where the second equality holds because $(r \otimes \top) \wedge-: A \longrightarrow A$ is a Q-module homomorphism. It remains to check that $(A, \otimes)$ is generated by $i$. This is easy, since for all $r \in \mathbf{Q}$ and $x \in A$,

$$
r \otimes x=r \otimes(x \wedge \top)=x \wedge(r \otimes \top)=x \wedge i(r) .
$$

Remark 3.7. Let $\mathrm{Q}=(\mathrm{Q}, \&)$ be a commutative and integral quantale, viewed as a $\mathrm{Q}$-module. It is trivial that $(\mathrm{Q}, \&)$ is spatial. But, the map

$$
r \wedge-:(\mathrm{Q}, \&) \longrightarrow(\mathrm{Q}, \&)
$$

is a $Q$-module homomorphism for all $r \in \mathrm{Q}$ if, and only if, the quantale $(\mathrm{Q}, \&)$ is a frame, i.e., $\&=\wedge$. Sufficiency is clear, to see the necessity, let $r \in Q$. Since $r \wedge-$ preserves the Q-action, it follows that

$$
r=r \wedge(r \& 1)=r \&(r \wedge 1)=r \& r
$$

hence $r$ is idempotent and consequently, $(\mathrm{Q}, \&)$ is a frame.

\section{Sobriety of $\mathcal{F}$-domains}

It is well-known in domain theory that the Scott topology of a domain (= continuous dcpo) is sober [3, 4. This section concerns an analogy of this conclusion in the enriched context when $\mathrm{Q}$ is an integral and commutative quantale. 
For each weight $\phi$ and each coweight $\psi$ of a Q-ordered set $X$, let

$$
\phi \pitchfork \psi=\bigvee_{x \in X} \phi(x) \& \psi(x)
$$

It is easily verified that for each weight $\phi$, each coweight $\psi$, and each point $a$ of a Q-ordered set $X$,

$$
\phi \pitchfork X(a,-)=\phi(a) \quad \text { and } \quad X(-, a) \pitchfork \psi=\psi(a) .
$$

Remark 4.1. From the viewpoint of fuzzy set theory, the value $\phi \pitchfork \psi$ measures the degree that $\phi$ and $\psi$ have a common point when $\phi$ and $\psi$ are viewed as fuzzy subsets of $X$. From the perspective of category theory, $\phi \pitchfork \psi$ is the colimit of $\psi: X \longrightarrow\left(\mathrm{Q}, \alpha_{L}\right)$ weighted by $\phi$, i.e., the supremum of $\psi \rightarrow(\phi)$ in $\left(\mathrm{Q}, \alpha_{L}\right)$ (c.f. [19, Example 2.11]). Furthermore, if we view $\phi$ as a fuzzy relation $X \longrightarrow *$ from $X$ to a singleton set and view $\psi$ as a fuzzy relation $* \nrightarrow X$ from a singleton set to $X$, then $\phi \pitchfork \psi$ is the composite fuzzy relation $\phi \circ \psi: * \longrightarrow X \longrightarrow *$.

Definition 4.2. ([19, 33, 34]) A flat ideal of a Q-ordered $X$ is a weight $\phi$ of $X$ that is (i) inhabited in the sense that $\bigvee_{x \in X} \phi(x) \geq k$; and (ii) flat in the sense that for any coweights $\psi_{1}, \psi_{2}$ of $X$,

$$
\phi \pitchfork\left(\psi_{1} \wedge \psi_{2}\right)=\left(\phi \pitchfork \psi_{1}\right) \wedge\left(\phi \pitchfork \psi_{1}\right) .
$$

Flat ideals are first introduced by Vickers in the case that $Q$ is Lawvere's quantale $\left([0, \infty]^{\mathrm{op}},+\right)$ and are called flat left modules [34]. Flat ideals provide a natural analogy of ideals (= directed lower sets of partially ordered sets) in the Q-valued context. We haste to remark that there exist different extensions of the notion of directed lower set to the enriched context, with flat ideal being one of them. A comparative study of some of these extensions can be found in [19].

Proposition 4.3. ([19, 33]) If $f: X \rightarrow Y$ preserves $Q$-order, then for each flat ideal $\phi$ of $X, f \rightarrow(\phi)$ is a flat ideal of $Y$.

For each Q-ordered set $X$, write

$$
\mathcal{F} X
$$

for the subset of $\mathcal{P} X$ consisting of flat ideals. It is obvious that for each $a \in X$, the weight $X(-, a)$ is a flat ideal of $X$, so the Yoneda embedding $\mathrm{y}_{X}: X \longrightarrow \mathcal{P} X$ factors through $\mathcal{F} X$. The assignment $X \mapsto \mathcal{F} X$ defines a saturated class of weights on Q-Ord, see [19, Proposition 4.5]. In other words, it gives rise to a submonad

$$
\mathbb{F}=(\mathcal{F}, \mathrm{m}, \mathrm{y})
$$

of the monad $\mathbb{P}=(\mathcal{P}, \mathrm{m}, \mathrm{y})$.

A Q-ordered set $X$ is $\mathcal{F}$-cocomplete if every flat ideal of $X$ has a supremum. It is clear that $X$ is $\mathcal{F}$-cocomplete if, and only if, the map

$$
\mathrm{y}_{X}: X \longrightarrow \mathcal{F} X, \quad x \mapsto X(-, x)
$$

has a left adjoint.

Separated and $\mathcal{F}$-cocomplete $Q$-ordered sets are precisely the algebras of the monad $\mathbb{F}=(\mathcal{F}, \mathrm{m}, \mathrm{y})$ (see e.g. [18, Section 3]) and provide an analogy of dcpos $(=$ directed 
complete partially ordered sets) [3, 4] in the Q-valued context. Such a Q-ordered set will be called an $\mathcal{F}$-dcpo. Since there exist different extensions of directed lower sets, hence different extensions of dcpos to the enriched context (see e.g. [19]), instead of the term "Q-dcpo", we adopt "F-depo" with the prefix $\mathcal{F}$ indicating that such a "Q-valued dсpo" is defined with respect to the class $\mathcal{F}$ of flat weights. This remark also applies to $\mathcal{F}$-domains defined below.

Proposition 4.4. The specialization $\mathrm{Q}$-order of a sober $\mathrm{Q}$-topological space is $\mathcal{F}$-cocomplete.

Proof. Let $X$ be a sober Q-topological space. We show that every flat ideal $\phi$ in $\Omega X$ has a supremum. Since each open set of $X$ is a coweight of $\Omega X$, it is readily verified that

$$
p: \mathcal{O}(X) \longrightarrow \mathrm{Q}, \quad p(\lambda)=\phi \pitchfork \lambda
$$

is a point of the Q-module $\mathcal{O}(X)$. Thus, there is some $a$ of $X$ such that $p(\lambda)=\lambda(a)$ for all $\lambda \in \mathcal{O}(X)$. We claim that $a$ is a supremum of $\phi$. Indeed, for all $b \in X$,

$$
\begin{aligned}
\Omega X(a, b) & =\bigwedge_{\lambda \in \mathcal{O}(X)} \lambda(a) \rightarrow \lambda(b) \\
& =\bigwedge_{\lambda \in \mathcal{O}(X)}\left(\bigvee_{x \in X} \phi(x) \& \lambda(x) \rightarrow \lambda(b)\right) \\
& =\bigwedge_{x \in X}\left(\phi(x) \rightarrow \bigwedge_{\lambda \in \mathcal{O}(X)}(\lambda(x) \rightarrow \lambda(b))\right) \\
& =\operatorname{sub}_{X}(\phi, \Omega X(-, b)),
\end{aligned}
$$

hence $a$ is a supremum of $\phi$ in $\Omega X$.

Definition 4.5. A Q-ordered set $X$ is an $\mathcal{F}$-domain if $X$ is separated and there exists a string of adjunctions

$$
\downarrow \dashv \sup \dashv \mathrm{y}_{X}: X \longrightarrow \mathcal{F} X \text {. }
$$

Said differently, a Q-ordered set $X$ is an $\mathcal{F}$-domain if it is an $\mathcal{F}$-dcpo and is continuous in the sense that for each $x \in X$, there is a flat ideal $\downarrow x$ such that for all $\phi \in \mathcal{F} X$,

$$
\operatorname{sub}_{X}(\downarrow x, \phi)=X(x, \sup \phi) .
$$

When flat ideals are taken as directed lower sets in the $\mathrm{Q}$-valued context, $\mathcal{F}$-domains are then an analogy of domains (= continuous dcpos) [3, 4].

Example 4.6. For each $\mathrm{Q}$-ordered set $X$, the set $\mathcal{F} X$ of flat ideas in $X$ with the inclusion Q-order is an $\mathcal{F}$-domain. This is an instance of [18, Proposition 3.3] or [19, Theorem 4.1]. Actually, for each $\mathrm{Q}$-ordered set $X$ we have a string of adjunctions

$$
\mathrm{y}_{X} \rightarrow \mathrm{y}_{X}^{\overleftarrow{ }} \dashv \mathrm{y}_{\mathcal{F} X}: \mathcal{F} X \longrightarrow \mathcal{F F} X
$$

where $\mathrm{y}_{X}: X \longrightarrow \mathcal{F} X$ is the unit of the monad $\mathbb{F}=(\mathcal{F}, \mathrm{m}, \mathrm{y})$, hence $\mathcal{F} X$ is an $\mathcal{F}$-domain. In particular, the supremum of a flat ideal $\Phi$ of $\mathcal{F} X$ is given by

$$
\sup \Phi=\mathrm{y}_{X}^{\overleftarrow{X}}(\Phi)=\bigvee_{\phi \in \mathcal{F} X} \Phi(\phi) \& \phi
$$


Remark 4.7 (When $Q$ is a frame, III). Assume that $Q=(Q, \&)$ is a frame, i.e., $\&=\wedge$. It follows from [33, Proposition 3.11] that for each Q-ordered set $X$, a weight $\phi$ is a flat ideal if, and only if, it is inhabited and

$$
\phi(x) \wedge \phi(y)=\bigvee_{z \in X} \phi(z) \wedge X(x, z) \wedge X(y, z)
$$

for all $x, y \in X$. So, in this case, a flat ideal of $X$ is precisely an ideal of $X$ in the sense of [16, Definition 5.1]; the fuzzy dcpos and fuzzy domains studied in [21, 36, 38, 39] are $\mathcal{F}$-cocomplete $\mathrm{Q}$-ordered sets and $\mathcal{F}$-domains, respectively.

Let $X$ be an $\mathcal{F}$-cocomplete $\mathrm{Q}$-ordered set. The way below $\mathrm{Q}$-relation (relative to flat ideals) on $X$ refers to the Q-relation $w: X \times X \longrightarrow \mathrm{Q}$ defined by

$$
w(x, y)=\bigwedge_{\phi \in \mathcal{F} X} X(y, \sup \phi) \rightarrow \phi(x) .
$$

We list below some basic properties of the way below Q-relation $w$, they are an instance of the properties of quantitative domains based on a class of weights, see e.g. [7, 8, 35]. For all $x, y, z, u \in X$,

(i) $w(x, y) \leq X(x, y)$;

(ii) $w(y, z) \& X(x, y) \leq w(x, z)$, in particular, $w(-, z)$ is a weight of $X$;

(iii) $X(z, u) \& w(y, z) \leq w(y, u)$, in particular, $w(y,-)$ is a coweight of $X$.

(iv) If $X$ is an $\mathcal{F}$-domain, then $w$ is interpolative in the sense that

$$
w(x, y)=\bigvee_{z \in X} w(z, y) \& w(x, z) .
$$

(v) If $X$ is a separated Q-ordered set of which every flat ideal has a supremum, then $X$ is a $\mathcal{F}$-domain if, and only if, for all $x \in X$, the weight $w(-, x)$ is a flat ideal with supremum $x$. In this case, the left adjoint $\downarrow: X \longrightarrow \mathcal{F} X$ of sup: $\mathcal{F} X \longrightarrow X$ is given by $\downarrow x=w(-, x)$.

Example 4.8 (When $Q$ is a frame, IV). Assume that $Q=(Q, \&)$ is a frame, i.e., $\&=\wedge$. Then $Q$ together with the canonical $Q$-order is an $\mathcal{F}$-domain; that is, $\left(\mathrm{Q}, \alpha_{L}\right)$ is an $\mathcal{F}$ domain. By [40, Example 3.14] and Example 4.7, the assignment $x \mapsto x \vee(\mathrm{id} \rightarrow 0)$ defines a left adjoint of the map that sends each flat ideal of $\left(\mathrm{Q}, \alpha_{L}\right)$ to its supremum. Thus, $\left(\mathrm{Q}, \alpha_{L}\right)$ is an $\mathcal{F}$-domain. The way below $\mathrm{Q}$-relation on $\left(\mathrm{Q}, \alpha_{L}\right)$ is given by $w(y, x)=x \vee(y \rightarrow 0)$.

For a general quantale $\mathrm{Q},\left(\mathrm{Q}, \alpha_{L}\right)$ may fail to be an $\mathcal{F}$-domain, see Theorem 5.3 below.

Definition 4.9. ([19]) A fuzzy set $\psi: X \longrightarrow \mathrm{Q}$ of a $\mathrm{Q}$-ordered set $X$ is Scott open if it is a coweight and

$$
\psi(\sup \phi) \leq \phi \pitchfork \psi
$$

for each flat ideal $\phi$ of $X$ whenever $\sup \phi$ exists.

Since for any weight $\phi$ and any coweight $\psi$ of $X$,

$$
k \leq X(\sup \phi, \sup \phi)=\mathcal{P} X(\phi, X(-, \sup \phi))
$$


whenever $\sup \phi$ exists, it follows that

$$
\phi(x) \leq X(x, \sup \phi) \leq \psi(x) \rightarrow \psi(\sup \phi)
$$

for all $x \in X$, hence $\phi \pitchfork \psi \leq \psi(\sup \phi)$. Therefore, the inequality in Definition 4.9] is actually an equality.

Given a $\mathrm{Q}$-ordered set $X$, the family of Scott open fuzzy sets form a Q-topology on $X$ [19, Proposition 5.2], called the Scott Q-topology on $X$ and denoted by $\sigma(X)$. We write

\section{$\Sigma X$}

for the Q-topological space $(X, \sigma(X))$.

Now we state the main result of this section.

Theorem 4.10. Let $\mathrm{Q}$ be an integral and commutative quantale. Then the Scott $\mathrm{Q}$ topology of each $\mathcal{F}$-domain is sober.

Lemma 4.11. Let $X$ be an $\mathcal{F}$-domain and let $\psi: X \longrightarrow\left(\mathrm{Q}, \alpha_{L}\right)$ be a $\mathrm{Q}$-order-preserving map, viewed as a fuzzy subset of $X$. Then the interior of $\psi$ in $\Sigma X$ is given by

$$
\psi^{\circ}=\bigvee_{a \in X} w(a,-) \& \psi(a)
$$

Proof. First, making use of the interpolation property of the way below Q-relation, one sees that for each $x \in X$, the fuzzy set $w(x,-): X \longrightarrow \mathrm{Q}$ is Scott open.

Next, we show that $\psi$ is open if, and only if,

$$
\psi=\bigvee_{a \in X} w(a,-) \& \psi(a)
$$

The conclusion follows from this fact immediately. Sufficiency is clear since each $w(a,-)$ is open. To see the necessity, we check that for all $x \in X$,

$$
\psi(x)=\bigvee_{a \in X} w(a, x) \& \psi(a)
$$

Since $X$ is an $\mathcal{F}$-domain, $w(-, x)$ is a flat ideal with $x$ as a supremum. Since $\psi$ is Scott open, then

$$
\psi(x)=\psi(\sup w(-, x))=w(-, x) \pitchfork \psi=\bigvee_{a \in X} w(a, x) \& \psi(a)
$$

Proof of Theorem 4.10. Write $\mathcal{O}(X)$ for the set of open sets of the Q-topological space $\Sigma X$, viewed as a Q-module. Let $p: \mathcal{O}(X) \longrightarrow \mathrm{Q}$ be a point of $\mathcal{O}(X)$. We need to show that there is a unique element $b$ of $X$ such that for each open set $\psi$ of $\Sigma X, p(\psi)=\psi(b)$.

Define $\phi: X \longrightarrow \mathrm{Q}$ by

$$
\phi(a)=p(w(a,-)) .
$$

We claim that $\phi$ is a flat ideal and the supremum of $\phi$ satisfies the requirement.

Step 1. $\phi$ is an inhabited weight of $X$. Since $Q$ is integral, the top element 1 of $Q$ is the unit of \&, then

$$
1_{X}=1_{X}^{\circ}=\bigvee_{a \in X} w(a,-) \& 1=\bigvee_{a \in X} w(a,-)
$$


thus

$$
\bigvee_{a \in X} \phi(a)=\bigvee_{a \in X} p\left((w(a,-))=p\left(\bigvee_{a \in X} w(a,-)\right)=p\left(1_{X}\right)=1\right.
$$

which shows that $\phi$ is inhabited. That $\phi$ is a weight follows from that for all $a, b \in X$,

$$
\phi(b) \& X(a, b)=p(w(b,-)) \& X(a, b)=p(w(b,-) \& X(a, b)) \leq p(w(a,-))=\phi(a) .
$$

Step 2. $\phi$ is flat. Since for each coweight $\psi$ of $X$,

$$
\begin{aligned}
\phi \pitchfork \psi & =\bigvee_{a \in X} p(w(a,-)) \& \psi(a) \\
& =\bigvee_{a \in X} p(w(a,-) \& \psi(a)) \\
& =p\left(\bigvee_{a \in X} w(a,-) \& \psi(a)\right) \\
& =p\left(\psi^{\circ}\right),
\end{aligned}
$$

it follows that for any coweights $\psi_{1}, \psi_{2}$ of $X$,

$$
\begin{aligned}
\phi \pitchfork\left(\psi_{1} \wedge \psi_{2}\right) & =p\left(\left(\psi_{1} \wedge \psi_{2}\right)^{\circ}\right) \\
& =p\left(\psi_{1}^{\circ}\right) \wedge p\left(\psi_{2}^{\circ}\right) \\
& =\left(\phi \pitchfork \psi_{1}\right) \wedge\left(\phi \pitchfork \psi_{2}\right),
\end{aligned}
$$

hence $\phi$ is flat.

Step 3. Let $b$ be the supremum of the flat ideal $\phi$. Then for each open set $\psi$ of $\Sigma X$,

$$
\begin{aligned}
p(\psi) & =p\left(\bigvee_{a \in X} w(a,-) \& \psi(a)\right) \\
& =\bigvee_{a \in X} p(w(a,-) \& \psi(a)) \\
& =\bigvee_{a \in X} \phi(a) \& \psi(a) \\
& =\phi \pitchfork \psi \\
& =\psi(\sup \phi) \\
& =\psi(b) .
\end{aligned}
$$

The proof is completed.

Example 4.12 (When $Q$ is a frame, V). ([39, Theorem 3.5]) Assume that $Q=(Q, \&)$ is a frame, i.e., $\&=\wedge$. By Example 4.8, $\left(\mathrm{Q}, \alpha_{L}\right)$ is an $\mathcal{F}$-domain and $w(x, y)=y \vee(x \rightarrow 0)$ for all $x, y \in \mathrm{Q}$. Then it follows from Lemma 4.11 that $\Sigma\left(\mathrm{Q}, \alpha_{L}\right)$ coincides with the Sierpiński space $\mathbb{S}$, hence $\mathbb{S}=\Sigma \Omega \mathbb{S}$ in this case. But, this is not true for a general quantale, see Proposition 5.9 in the next section.

Remark 4.13. In the case that the quantale $Q$ is a frame, Theorem 4.10 and Proposition 4.4 are first proved in 38 . 


\section{Some examples in the case that $\mathrm{Q}=([0,1], \&)$}

The aim of this section is to present some examples of $\mathcal{F}$-domains and sober Q-topological spaces when $Q$ is the interval $[0,1]$ endowed with a continuous t-norm. In particular, a necessary and sufficient condition is obtained for $[0,1]$ together with the canonical Q-order to be an $\mathcal{F}$-domain.

A left-continuous $t$-norm on $[0,1]\left[13\right.$ is a map $\&:[0,1]^{2} \longrightarrow[0,1]$ that makes $([0,1], \&)$ into a commutative and integral quantale. A left-continuous t-norm that is continuous with respect to the usual topology is called a continuous t-norm [13]. Continuous t-norms play a decisive role in the BL-logic of Hájek [6].

Basic continuous t-norms and their implication operators are listed below:

(1) The Gödel t-norm:

$$
x \& y=\min \{x, y\} ; \quad x \rightarrow y= \begin{cases}1, & x \leq y, \\ y, & x>y\end{cases}
$$

The implication operator $\rightarrow$ of the Gödel t-norm is continuous except at $(x, x)$, $x<1$.

(2) The product t-norm:

$$
x \&_{P} y=x y ; \quad x \rightarrow y= \begin{cases}1, & x \leq y, \\ y / x, & x>y .\end{cases}
$$

The implication operator $\rightarrow$ of the product t-norm is continuous except at $(0,0)$.

(3) The Łukasiewicz t-norm:

$$
x \&_{£} y=\max \{0, x+y-1\} ; \quad x \rightarrow y=\min \{1-x+y, 1\} .
$$

The implication operator $\rightarrow$ of the Eukasiewicz t-norm is continuous on $[0,1]^{2}$.

Let \& be a continuous t-norm. An element $p \in[0,1]$ is idempotent if $p \& p=p$.

Proposition 5.1. ([13, Proposition 2.3]) Let \& be a continuous t-norm on $[0,1]$ and $p$ be an idempotent element of \&. Then $x \& y=x \wedge y$ whenever $x \leq p \leq y$.

It follows immediately that $y \rightarrow x=x$ whenever $x<p \leq y$ for some idempotent $p$. Another consequence of Proposition 5.1 is that for any idempotent elements $p, q$ with $p<q$, the restriction of \& to $[p, q]$, which is also denoted by \&, makes $[p, q]$ into a commutative quantale with $q$ being the unit element.

A continuous t-norm on $[0,1]$ is said to be Archimedean if it has no idempotent element other than 0 and 1. It is well-known that if $\&$ is a continuous Archimedean t-norm, then the quantale $([0,1], \&)$ is either isomorphic to $\left([0,1], \&_{£}\right)$ or to $\left([0,1], \&_{P}\right)$, see e.g. [13, 23].

Theorem 5.2. ([13, 23]) Let \& be a continuous t-norm. Then for each non-idempotent element $c$ of $([0,1], \&)$, there exist idempotent elements $c^{-}$and $c^{+}$such that $c^{-}<c<c^{+}$ and the quantale $\left(\left[c^{-}, c^{+}\right], \&\right)$ is either isomorphic to $\left([0,1], \&_{E}\right)$ or to $\left([0,1], \&_{P}\right)$.

Proposition 5.3. Let \& be a continuous t-norm and let $\mathrm{Q}=([0,1], \&)$. Then, the following are equivalent: 
(1) The $\mathrm{Q}$-ordered set $\left([0,1], \alpha_{L}\right)$ is an $\mathcal{F}$-domain.

(2) For each non-idempotent element $c \in[0,1]$, the quantale $\left(\left[c^{-}, c^{+}\right], \&\right)$ is isomorphic to $\left([0,1], \&_{P}\right)$ whenever $c^{-}>0$.

In order to prove the conclusion, we present four lemmas first.

Lemma 5.4. Let \& be a continuous Archimedean t-norm and let $\mathrm{Q}=([0,1], \&)$. Then $\phi:[0,1] \longrightarrow[0,1]$ is a flat ideal of the $\mathrm{Q}$-ordered set $\left([0,1], \alpha_{L}\right)$ if, and only if, $\phi=\alpha_{L}(-, a)$ for some $a \in[0,1]$.

Proof. A direct consequence of Corollary 3.14 and Theorem 3.18 in [19].

Lemma 5.5. Let \& be a continuous t-norm and let $\mathrm{Q}=([0,1], \&)$. Let $\phi$ be a flat ideal of $\left([0,1], \alpha_{L}\right)$. If $c \in[0,1]$ is idempotent and $c \leq \phi(c)$, then $\phi(c)$ is idempotent.

Proof. Consider the following coweights of $\left([0,1], \alpha_{L}\right): \psi_{1} \equiv \phi(c)$ and $\psi_{2}=\alpha_{L}(c,-)$.

Since $\phi \pitchfork \psi_{1}=\phi(c)=\phi \pitchfork \psi_{2}$ and

$$
\left(\psi_{1} \wedge \psi_{2}\right)(x)= \begin{cases}c \rightarrow x, & 0 \leq x<c \\ \phi(c), & c \leq x \leq 1\end{cases}
$$

it follows that

$$
\begin{aligned}
\phi(c) & =\phi \pitchfork\left(\psi_{1} \wedge \psi_{2}\right) \\
& =\left(\bigvee_{x \in[0, c)} \phi(x) \&(c \rightarrow x)\right) \vee\left(\bigvee_{x \in[c, 1]} \phi(x) \& \phi(c)\right) \\
& =\left(\bigvee_{x \in[0, c)}(c \rightarrow x)\right) \vee(\phi(c) \& \phi(c)) \quad(\forall x \in[0, c), c \rightarrow x \leq c \leq \phi(c) \leq \phi(x)) \\
& \leq c \vee(\phi(c) \& \phi(c)) \\
& =\phi(c) \& \phi(c), \quad(c \leq \phi(c))
\end{aligned}
$$

hence $\phi(c)$ is idempotent.

For a non-idempotent element $c$ in $([0,1], \&)$, write $\mathrm{Q}^{c}$ for the quantale obtained by restricting \& to $\left[c^{-}, c^{+}\right]$; that is,

$$
\mathrm{Q}^{c}=\left(\left[c^{-}, c^{+}\right], \&\right)
$$

Lemma 5.6. Let \& be a continuous t-norm, let $\mathrm{Q}=([0,1], \&)$, and let $\phi$ be a flat ideal of $\left([0,1], \alpha_{L}\right)$. If $c \in[0,1]$ is non-idempotent and $\sup \phi \geq c$, then the map

$$
\rho:\left[c^{-}, c^{+}\right] \longrightarrow\left[c^{-}, c^{+}\right], \quad \rho(t)=c^{+} \wedge \phi(t)
$$

is a flat ideal of the $\mathrm{Q}^{c}$-ordered set $\left(\left[c^{-}, c^{+}\right], \alpha_{L}^{c}\right)$, where $\alpha_{L}^{c}$ refers to the canonical $\mathrm{Q}^{c}$-order on $\left[c^{-}, c^{+}\right]$.

Proof. Since $\phi\left(c^{+}\right) \geq \phi(1)=\sup \phi=c>c^{-}$, it follows that $\phi(x) \geq c^{-}$for all $x \in\left[c^{-}, c^{+}\right]$, hence $\rho$ is well-defined. That $\rho$ is a weight of $\left(\left[c^{-}, c^{+}\right], \alpha_{L}^{c}\right)$ is clear, it remains to check that it is inhabited and flat.

Step 1. Since $\phi\left(c^{-}\right)>c^{-}$, it follows from Lemma 5.5 that $\phi\left(c^{-}\right)$is idempotent, hence $\phi\left(c^{-}\right) \geq c^{+}$and $\rho\left(c^{-}\right)=c^{+}$, which shows that $\rho$ is inhabited. 
Step 2. For each coweight $\psi$ of $\left(\left[c^{-}, c^{+}\right], \alpha_{L}^{c}\right)$, the map $\widetilde{\psi}:[0,1] \longrightarrow[0,1]$, given by

$$
\widetilde{\psi}(x)= \begin{cases}x, & x \in\left[0, c^{-}\right), \\ \psi(x), & x \in\left[c^{-}, c^{+}\right], \\ \psi\left(c^{+}\right), & x \in\left(c^{+}, 1\right],\end{cases}
$$

is a coweight of $\left([0,1], \alpha_{L}\right)$. The verification is straightforward and omitted here.

Step 3. $\rho$ is flat; that is, for any coweights $\psi_{1}, \psi_{2}$ of $\left(\left[c^{-}, c^{+}\right], \alpha_{L}^{c}\right)$,

$$
\rho \pitchfork\left(\psi_{1} \wedge \psi_{2}\right)=\left(\rho \pitchfork \psi_{1}\right) \wedge\left(\rho \pitchfork \psi_{2}\right) .
$$
Thus,

First, a routine calculation shows that $\rho \pitchfork \psi=\phi \pitchfork \widetilde{\psi}$ for all coweight $\psi$ of $\left(\left[c^{-}, c^{+}\right], \alpha_{L}^{c}\right)$.

$$
\begin{array}{rlr}
\rho \pitchfork\left(\psi_{1} \wedge \psi_{2}\right) & =\phi \pitchfork\left(\widetilde{\psi_{1} \wedge \psi_{2}}\right) \\
& =\phi \pitchfork\left(\widetilde{\psi_{1}} \wedge \widetilde{\psi_{2}}\right) \\
& =\left(\phi \pitchfork \widetilde{\psi_{1}}\right) \wedge\left(\phi \pitchfork \widetilde{\psi_{2}}\right) & \quad(\phi \text { is flat }) \\
& =\left(\rho \pitchfork \psi_{1}\right) \wedge\left(\rho \pitchfork \psi_{2}\right),
\end{array}
$$

which completes the proof.

Lemma 5.7. Let \& be a continuous t-norm and let $\mathrm{Q}=([0,1], \&)$. For each $x \in[0,1]$, define $d(x):[0,1] \longrightarrow[0,1]$ by

$$
d(x)(t)= \begin{cases}t \rightarrow 0, & x=0, \\ 1, & x>0, t=0, \\ x^{+} \wedge(t \rightarrow x), & x>0, t>0 .\end{cases}
$$

Then $d(x)$ is the smallest flat ideal of $\left([0,1], \alpha_{L}\right)$ with supremum larger than or equal to $x$.

Proof. The verification of that $d(x)$ is a flat ideal with supremum $x$ is straightforward. Now we check that for any flat ideal $\phi$ of $\left([0,1], \alpha_{L}\right)$ with $\sup \phi \geq x$, it holds that $d(x) \leq \phi$. The conclusion is obvious if $x$ is idempotent. Assume that $x$ is not idempotent. By Lemma 5.6, the map

$$
\rho:\left[x^{-}, x^{+}\right] \longrightarrow\left[x^{-}, x^{+}\right], \quad \rho(t)=x^{+} \wedge \phi(t)
$$

is a flat ideal of the $\mathrm{Q}^{x}$-ordered set $\left(\left[x^{-}, x^{+}\right], \alpha_{L}^{x}\right)$. Since the quantale $\left(\left[x^{-}, x^{+}\right], \&\right)$ is isomorphic to $[0,1]$ endowed with a continuous Archimedean t-norm, it follows from Lemma 5.4 that there is some $b \in\left[x^{-}, x^{+}\right]$such that

$$
\rho(t)=\alpha_{L}^{x}(t, b)=x^{+} \wedge(t \rightarrow b)
$$

for all $t \in\left[x^{-}, x^{+}\right]$.

We claim that $b \geq x$, hence $d(x)(t) \leq \rho(t) \leq \phi(t)$ for all $t \in\left[x^{-}, x^{+}\right]$. Suppose on the contrary that $b<x$. Take some $z \in\left(x, x^{+}\right)$such that $z \rightarrow b<x$. Then $x>\rho(z)=$ $x^{+} \wedge \phi(z)$, hence $\phi(z)=\rho(z)<x$, contradicting that $\phi(z) \geq \phi(1)=\sup \phi \geq x$ (the equality holds by Example 2.6).

Finally, since

$$
d(x)(t)=x^{+}=\rho\left(x^{-}\right) \leq \phi(t)
$$

for all $t \in\left(0, x^{-}\right)$and

$$
d(x)(t)=x \leq \phi(1) \leq \phi(t)
$$

for all $t \in\left(x^{+}, 1\right]$, it follows that $d(x) \leq \phi$, as desired. 
Proof of Proposition [5.3. Write $X$ for the Q-ordered set $\left([0,1], \alpha_{L}\right)$ and for each $x \in X$, let $d(x)$ be the smallest flat ideal of $X$ with supremum larger than or equal to $x$, as given in Lemma 5.7. Then, by Theorem 2.3, it is sufficient to show that the map $d: X \longrightarrow \mathcal{F} X$ preserves Q-order if, and only if, \& satisfies the condition stated in (2). We check the only-if-part here and leave the if-part to the reader.

Suppose on the contrary that \& does not satisfy that condition. Then there exist idempotent elements $p, q>0$ such that the restriction of \& on $[p, q]$ is isomorphic to the Łukasiewicz t-norm. Pick $t \in(p, q)$. Then

$$
\begin{aligned}
X(t, p) & =t \rightarrow p \\
& >q \rightarrow p \\
& \geq \bigwedge_{x \in(0,1]}(q \wedge(x \rightarrow t) \rightarrow p \wedge(x \rightarrow p)) \\
& =\mathcal{F} X(d(t), d(p)),
\end{aligned}
$$

which contradicts that $d: X \longrightarrow \mathcal{F} X$ preserves $\mathrm{Q}$-order.

Proposition 5.8. Let \& be a continuous $t$-norm on $[0,1]$ and let $Q=([0,1], \&)$. If for each non-idempotent element $c \in[0,1]$, the quantale $\left(\left[c^{-}, c^{+}\right], \&\right)$ is isomorphic to $\left([0,1], \&_{P}\right)$ whenever $c^{-}>0$, then the Q-topological space $\Sigma\left([0,1], \alpha_{L}\right)$ is sober.

Proof. This follows from a combination of Proposition 5.3 and Theorem 4.10.

A celebrated result of Scott [28] says that if $X$ is an injective $T_{0}$ topological space, then the topology of $X$ is precisely the Scott topology of its specialization order. It is shown in [39] that this is also true in the $Q$-enriched setting if the quantale $Q$ is a frame. But, this is not true in general, as we see below.

Proposition 5.9. Let \& be a continuous t-norm and let $\mathrm{Q}=([0,1], \&)$. Let $\mathbb{S}$ be the Sierpiński Q-topological space. Then $\mathbb{S}=\Sigma \Omega \mathbb{S}$ if, and only if, \& is the Gödel t-norm.

A lemma first.

Lemma 5.10. Let \& be a continuous t-norm and let $\mathrm{Q}=([0,1], \&)$. Then a coweight $\psi$ of $\left([0,1], d_{L}\right)$ is Scott open if, and only if, for all $x \in(0,1], \psi(x)>x^{+}$implies $\psi(x)=\psi(0)$.

Proof. This is the content of [43, Lemma 4.11]. We present here a simple proof with help of Lemma 5.7. For each $x \in[0,1]$, let $d(x)$ be the smallest flat ideal of $X$ with supremum larger than or equal to $x$. For each coweight $\psi$ of $\left([0,1], d_{L}\right)$, since we always have

$$
\psi(0)=d(0)(0) \& \psi(0) \leq d(0) \pitchfork \psi,
$$

it follows that $\psi$ is Scott open if, and only if, for all $x \in(0,1], \psi(x) \leq d(x) \pitchfork \psi$. Because

$$
\begin{aligned}
d(x) \pitchfork \psi & =\psi(0) \vee \bigvee_{t>0}\left(x^{+} \wedge(t \rightarrow x)\right) \& \psi(t) \\
& =\psi(0) \vee \bigvee_{t>0} x^{+} \&(t \rightarrow x) \& \psi(t) \quad\left(x^{+}\right. \text {is idempotent, Proposition 5.1) } \\
& =\psi(0) \vee\left(x^{+} \wedge \psi(x)\right),
\end{aligned}
$$

therefore, $\psi$ is Scott open if, and only if, for each $x \in(0,1], \psi(x)>x^{+} \operatorname{implies} \psi(x)=$ $\psi(0)$. 
Proof of Proposition 5.9. Sufficiency follows from Example 4.12. To see the necessity, suppose on the contrary that $([0,1], \&)$ has a non-idempotent element, say $c$. Then the map

$$
\lambda(x):=\left(\left(c \rightarrow c^{-}\right) \vee(c \rightarrow x)\right) \wedge c^{+}
$$

satisfies the conditions in Lemma 5.10, hence it is a Scott open set of $\Omega \mathbb{S}$. But, $\lambda$ is not an open set of $\mathbb{S}$.

Since for each commutative and unital quantale $Q$, the space $\mathbb{S}$ is an injective Qtopological space, Proposition [5.9 shows that in the quantale-enriched context, the Qtopology of an injective Q-topological space may not be the Scott Q-topology of its specialization Q-order.

We end this section with a discussion of the sobriety of the space $\Sigma\left([0,1], \alpha_{R}\right)$, where $\alpha_{R}$ is the opposite of the canonical Q-order on $[0,1]$.

Lemma 5.11. ([43, Lemma 4.14]) Let \& be a continuous t-norm and let $\mathrm{Q}=([0,1], \&)$. Then, for each coweight $\psi$ of $\left([0,1], \alpha_{R}\right)$, it holds that

(i) if $\psi(x) \leq x^{-}$then $\psi(x)=\psi(1)$;

(ii) if $x$ is idempotent and $\psi(x) \geq x$ then $\psi(1) \geq x$.

Lemma 5.12. ([43, Lemma 4.15]) Let \& be a continuous $t$-norm and let $Q=([0,1], \&)$. If $b$ is a nontrivial idempotent element in $([0,1], \&)$, then for each Scott open set $\psi$ of $\left([0,1], \alpha_{R}\right)$, either $\psi$ is a constant map or $\psi(x) \leq b$ for all $x \in[0,1]$.

Proposition 5.13. Let \& be a continuous t-norm and let $\mathrm{Q}=([0,1], \&)$. Then the following are equivalent:

(1) The $\mathrm{Q}$-ordered set $\left([0,1], \alpha_{R}\right)$ is an $\mathcal{F}$-domain.

(2) The Q-topological space $\Sigma\left([0,1], \alpha_{R}\right)$ is sober.

(3) \& is Archimedean.

Proof. Write $X$ for the Q-ordered set $\left([0,1], \alpha_{R}\right)$.

$(1) \Rightarrow(2)$ Theorem 4.10 ,

$(2) \Rightarrow(3)$ Suppose on the contrary that $([0,1], \&)$ has a nontrivial idempotent element, say $b$. By Lemma 5.11 and Lemma 5.12, for all open set $\psi$ of $\Sigma X$ and all $x, y>b$, it holds that $\psi(x)=\psi(y)$. Therefore, $\Sigma X$ is not a $T_{0}$ space, hence not sober, contradicting the assumption.

$(3) \Rightarrow(1)$ By Corollary 3.14 and Theorem 3.18 in [19], a weight $\phi$ of $X$ is a flat ideal if, and only if, $\phi=X(-, a)$ for some $a \in[0,1]$. By help of this fact, one readily verifies that $a \mapsto X(-, a)$ defines a left adjoint of sup: $\mathcal{F} X \longrightarrow X$.

\section{References}

[1] F. Borceux, Handbook of Categorical Algebra, Vol. 2, Cambridge University Press, Cambridge, 1994.

[2] P. Eklund, J. Gutiérrez García, U. Höhle, J. Kortelainen, Semigroups in Complete Lattices. Quantales, Modules and Related Topics, Springer, 2018.

[3] G. Gierz, K.H. Hofmann, K. Keimel, J.D. Lawson, M. Mislove, D.S. Scott, Continuous Lattices and Domains, Cambridge University Press, Cambridge, 2003. 
[4] J. Goubault-Larrecq, Non-Hausdorff Topology and Domain Theory, Cambridge University Press, Cambridge, 2013.

[5] J. Gutiérrez García, U. Höhle, M.A. de Prada Vicente, On lattice-valued frames: The completely distributive case, Fuzzy Sets and Systems 161 (2010) 1022-1030.

[6] P. Hájek, Metamathematics of Fuzzy Logic, Kluwer Academic Publishers, Dordrecht, 1998.

[7] D. Hofmann, P. Waszkiewicz, Approximation in quantale-enriched categories, Topology and its Applications 158 (2011) 963-977.

[8] D. Hofmann, P. Waszkiewicz, A duality of quantale-enriched categories, Journal of Pure and Applied Algebra 216 (2012) 1866-1878.

[9] G. Jäger, W. Yao, Completely prime $L$-filters, irreducible $L$-filters and sobriety, Quaestiones Mathematicae 39 (2016) 831-844.

[10] P.T. Johnstone, Stone Spaces, Cambridge University Press, Cambridge, 1982.

[11] A. Joyal, M. Tierney, An Extension of the Galois Theory of Grothendieck, Memoirs of the American Mathematical Society, Vol. 51, No. 309. American Mathematical Society, Providence, Rhode Island, 1984.

[12] G.M. Kelly, V. Schmitt, Notes on enriched categories with colimits of some class, Theory and Applications of Categories 14 (2005) 399-423.

[13] E.P. Klement, R. Mesiar, E. Pap, Triangular Norms, Kluwer Academic Publishers, Dordrecht, 2000.

[14] W. Kotzé, Fuzzy sobriety and fuzzy Hausdorff, Quaestiones Mathematicae 20 (1997) 415-422.

[15] H. Lai, D. Zhang, Fuzzy preorder and fuzzy topology, Fuzzy Sets and Systems 157 (2006) 1865-1885.

[16] H. Lai, D. Zhang, Complete and directed complete $\Omega$-categories, Theoretical Computer Science 388 (2007) 1-25.

[17] H. Lai, D. Zhang, Concept lattices of fuzzy contexts: formal concept analysis vs. rough set theory, International Journal of Approximate Reasoning 50 (2009) 695707 .

[18] H. Lai, D. Zhang, Completely distributive enriched categories are not always continuous, Theory and Application of Categories 35 (2020) 64-88.

[19] H. Lai, D. Zhang, G. Zhang, A comparative study of ideals in fuzzy orders, Fuzzy Sets and Systems 382 (2020) 1-28.

[20] F.W. Lawvere, Metric spaces, generalized logic, and closed categories, Rendiconti del Seminario Matématico e Fisico di Milano 43 (1973) 135-166.

[21] M. Liu, B. Zhao, Two Cartesian closed subcategories of fuzzy domains, Fuzzy Sets and Systems 238 (2014) 102-112.

[22] S. Mac Lane, Categories for the Working Mathematician, Graduate Texts in Mathematics, Vol. 5, Springer, New York, second edition, 1998.

[23] P.S. Mostert, A.L. Shields, On the structure of semigroups on a compact manifold with boundary, Annals of Mathematics 65 (1957) 117-143. 
[24] A. Pultr, S.E. Rodabaugh, Categorical theoretic aspects of chain-valued frames, Part I: Categorical and presheaf theoretic foundations, Fuzzy Sets and Systems 159 (2008) 501-528.

[25] A. Pultr, S.E. Rodabaugh, Categorical theoretic aspects of chain-valued frames, Part II: Applications to lattice-valued topology, Fuzzy Sets and Systems 159 (2008) 529-558.

[26] S.E. Rodabaugh, Point-set lattice-theoretic topology, Fuzzy Sets and Systems 40 (1991) 296-345.

[27] K.I. Rosenthal, Quantales and Their Applications, Longman, Essex, 1990.

[28] D.S. Scott, Continuous lattices, in: Toposes, Algebraic Geometry and Logic, Lecture Notes in Mathematics, vol. 274, pp. 97-136. Springer, Berlin, 1972.

[29] S.K. Singh, A.K. Srivastava, On $Q$-sobriety, Quaestiones Mathematicae 39 (2016) 179-188.

[30] A.K. Srivastava, A.S. Khastgir, On fuzzy sobriety, Information Sciences 110 (1998) 195-205.

[31] I. Stubbe, Categorical structures enriched in a quantaloid: categories, distributors and functors, Theory and Applications of Categories 14 (2005) 1-45.

[32] I. Stubbe, Categorical structures enriched in a quantaloid: tensored and cotensored categories, Theory and Applications of Categories 16 (2006) 283-306.

[33] Y. Tao, H. Lai, D. Zhang, Quantale-valued preorders: Globalization and cocompleteness, Fuzzy Sets and Systems 256 (2014) 236-251.

[34] S. Vickers, Localic completion of generalized metric spaces, Theory and Application of Categories 14 (2005) 328-356.

[35] P. Waszkiewicz, On domain theory over Girard quantales, Fundamenta Informaticae 92 (2009) 1-24.

[36] W. Yao, Quantitative domains via fuzzy sets: Part I: Continuity of fuzzy directed complete posets, Fuzzy Sets and Systems 161 (2010) 973-987.

[37] W. Yao, An approach to fuzzy frames via fuzzy posets, Fuzzy Sets and Systems 166 (2011) 75-89.

[38] W. Yao, A survey of fuzzifications of frames, the Papert-Papert-Isbell adjunction and sobriety, Fuzzy Sets and Systems 190 (2012) 63-81.

[39] W. Yao, A categorical isomorphism between injective fuzzy $T_{0}$-spaces and fuzzy continuous lattices, IEEE Transactions on Fuzzy Systems 24 (2016) 131-139.

[40] D. Zhang, An enriched category approach to many valued topology, Fuzzy Sets and Systems 158 (2007) 349-366.

[41] D. Zhang, Sobriety of quantale-valued cotopological spaces, Fuzzy Sets and Systems 350 (2018) 1-19.

[42] D. Zhang, Y. Liu, L-fuzzy version of Stone's representation theorem for distributive lattices, Fuzzy Sets and Systems 76 (1995) 259-270.

[43] D. Zhang, G. Zhang, Continuous triangular norm based fuzzy topology, Archive for Mathematical Logic 58 (2019) 915-942. 